\title{
Neuromagnetic Responses to Frequency-Tagged Sounds: A New Method to Follow Inputs from Each Ear to the Human Auditory Cortex during Binaural Hearing
}

\author{
Nobuya Fujiki, ${ }^{1,2}$ Veikko Jousmäki, ${ }^{1}$ and Riitta Hari ${ }^{1,3}$ \\ ${ }_{1}^{1}$ Brain Research Unit, Low Temperature Laboratory, Helsinki University of Technology, FIN-02015 HUT, Espoo, Finland, \\ 2Department of Otolaryngology-Head and Neck Surgery, Kyoto University Graduate School of Medicine, Sakyo-ku, \\ Kyoto, 606-8507, Japan, and ${ }^{3}$ Department of Clinical Neurophysiology, Helsinki University Central Hospital, FIN-00290 \\ Helsinki, Finland
}

Binaural cortical responses are mixtures of inputs from both ears. We introduce here a novel method that allows, for the first time, to selectively follow these inputs in humans up to the cortex during binaural hearing. We recorded neuromagnetic cortical responses to amplitude-modulated continuous tones, with different modulation frequencies at each ear. During binaural hearing, the left- and right-ear inputs competed strongly in both auditory cortices: the right-hemisphere responses were symmetrically suppressed, compared with monaural stimulation, for sounds of both ears, whereas the left-hemisphere responses were suppressed significantly more for ipsilateral than contralateral sounds, thereby intensifying the right-ear dominance of the left auditory cortex. This type of hemisphereand ear-selective information on cortical binaural interaction could have important applications in human auditory neuroscience.

Key words: magnetoencephalography; frequency tagging; binaural hearing; amplitude-modulated tone; right-ear dominance; binaural suppressive interaction; steady-state responses
In everyday situations, we perceive environmental sounds binaurally. Because each ear projects to both left and right auditory cortex, although with contralateral dominance (Reite et al., 1981; Hari and Mäkelä, 1988; Mäkelä et al., 1993; Pantev et al., 1998), the inputs from each ear are processed simultaneously in both hemispheres. The resulting responses in the auditory cortices are thus mixtures of inputs from the left and right ears, and there has been no means to find out which part of the response derives from either ear. Such information would, however, be required to unravel whether binaural interaction affects differently inputs from the two ears.

Strong suppressive binaural interaction at the human auditory cortex has been documented in several magnetoencephalographic (MEG) studies (Reite et al., 1981; Pantev et al., 1986; Tiihonen et al., 1989) that have also indicated that the strength of binaural interaction may differ to some extent depending on the response type (Tiihonen et al., 1989). In these recordings, binaural responses of the human auditory cortex were much smaller than the sum of responses to monaural left- and right-ear sounds and, at some time instants, even smaller than responses to contralateral sounds presented alone.

Binaural interaction has also been convincingly demonstrated in animals, with ear-specific excitations-inhibitions observed in the firing pattern of auditory-cortex neurons (Imig and Adrian, 1977; Reser et al., 2000). However, even such direct recordings have not resolved whether, at the population level, binaural sup-

\footnotetext{
Received July 9, 2001; revised Nov. 14, 2001; accepted Nov. 19, 2001.

This study has been financially supported by the Academy of Finland, the Sigrid Jusélius Foundation, and the Japan Society for the Promotion of Science.

Correspondence should be addressed to Dr. Nobuya Fujiki, Department of Otolaryngology-Head and Neck Surgery, Kyoto University Graduate School of Medicine, Sakyo-ku, Kyoto 606-8507, Japan. E-mail: fujiki@hs.m.kyoto-u.ac.jp. Copyright (C) 2002 Society for Neuroscience $0270-6474 / 02 / 220001-04 \$ 15.00 / 0$
}

pression would be asymmetric with respect to ipsilateral versus contralateral sounds. Given the strong hemispheric specialization of many human brain functions, it would also be interesting to find out whether the binaural interaction in the human auditory cortex would show some hemispheric differences.

To obtain such information, we "labeled" the inputs from both ears by tagging the stimuli with amplitude modulations (AMs) of different frequencies at each ear and by following cortical responses at the modulation frequencies. Associated with the selectivity of MEG to signals arising from the auditory cortices of the two hemispheres (Hari, 1990), we were able to obtain both hemisphere- and ear-selective information. Our approach is analogous to visual frequency tagging, which has been successfully used for examination of binocular interactions (Brown et al., 1999). Both multiple simultaneous stimuli (Lins and Picton, 1995) and AM-labeled different melodies (Patel and Balaban, 2000) have been used to elicit auditory steady-state responses. However, the frequency-tagging approach has never been used previously to assess contributions of the auditory inputs from each ear to binaural responses generated in the auditory cortices of the left and right hemispheres.

This article is published in The Journal of Neuroscience, Rapid Communications Section, which publishes brief, peerreviewed papers online, not in print. Rapid Communications are posted online approximately one month earlier than they would appear if printed. They are listed in the Table of Contents of the next open issue of JNeurosci. Cite this article as: JNeurosci, 2002, 22:RC205 (1-4). The publication date is the date of posting online at www.jneurosci.org.

http://www.jneurosci.org/cgi/content/full/6052 


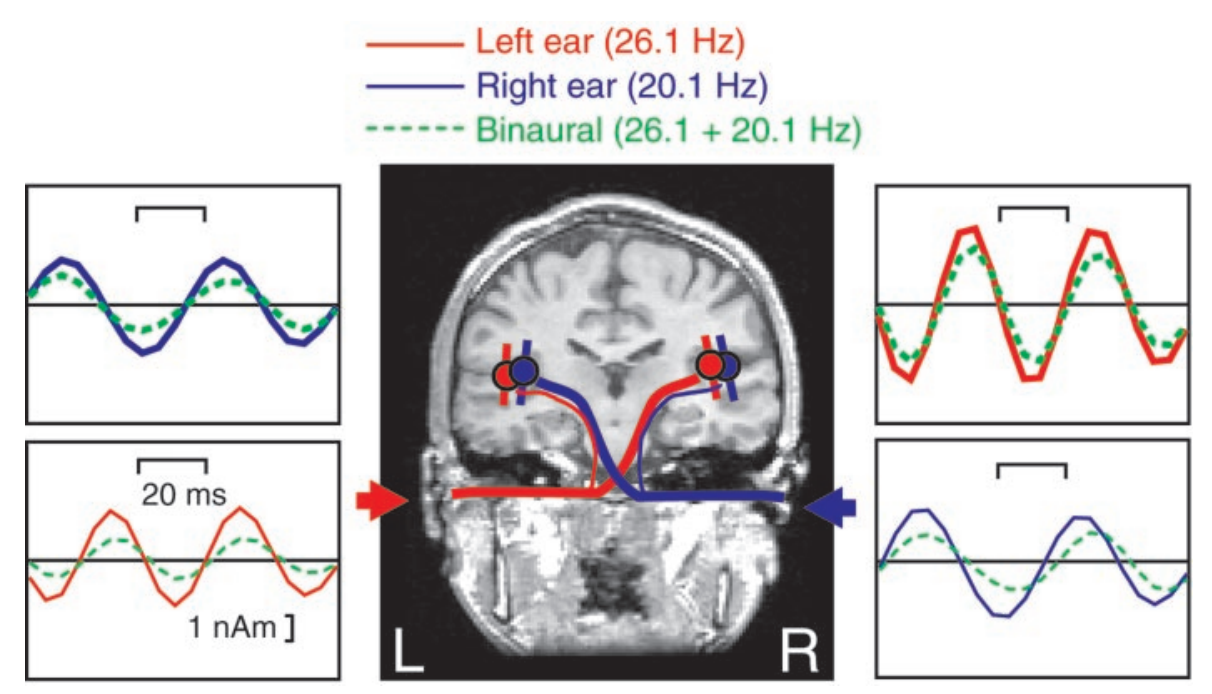

Figure 1. Steady-state fields in one representative subject. The middle shows schematically the central auditory pathways, with bilateral but contralaterally dominant cortical projections from each ear. The spheres show the sources of monaural SSFs in the supratemporal auditory cortices of both hemispheres, and the bars illustrate the associated source current directions. The sources have been superimposed on the subject's own magnetic resonance image. The traces illustrate the source strengths as a function of time for all stimuli, shown separately in both hemispheres. The color code of the stimuli and responses is given at the top.

Parts of this work have been published previously in abstract form (Fujiki et al., 2000).

\section{MATERIALS AND METHODS}

Subjects. We studied 12 normal-hearing right-handed subjects (five females, seven males; ages 24-38 years; mean \pm SD age of $29.5 \pm 5.0$ years). The study was approved by the Ethical Committee of the Helsinki Uusimaa Hospital District, and an informed consent was obtained from all subjects after full explanation of the procedure.

Stimulation. Continuous $1 \mathrm{kHz}$ tones were led through plastic tubes and earpieces in three different runs to the subject's left ear, right ear, or both. Different sinusoidal AMs, with $80 \%$ depth of modulation, were applied to the left- and right-ear tones: the left-ear tone was modulated at $26.1 \mathrm{~Hz}$, and the right ear tone was modulated at $20.1 \mathrm{~Hz}$. In a control study with six subjects, all of whom had also participated in the main study, the AM sides were reversed so that the left-ear tone was modulated at $20.1 \mathrm{~Hz}$ and the right-ear tone at $26.1 \mathrm{~Hz}$. The AM frequencies were selected on the basis of previous experience (Hari et al., 1989) and pilot experiments. We purposefully avoided AMs close to $40 \mathrm{~Hz}$ to minimize power-line contamination to the tiny steady-state fields (SSFs), and pilot experiments on a few subjects showed that reliable SSFs can be recorded at AMs $\sim 20-26 \mathrm{~Hz}$. We checked that the $1 \mathrm{kHz}$ rms power of the sound was the same within $1 \mathrm{~dB}$ with both $\mathrm{AM}$ frequencies.

Sound intensity was adjusted to the highest comfortable listening level, which was 40-60 $\mathrm{dB}$ above the individual sensation threshold. The subjects were instructed to attend the stimuli and to keep their eyes open.

Recording. Neuromagnetic cortical signals were recorded with a wholescalp neuromagnetometer (Vectorview; Neuromag Ltd., Helsinki, Finland), which has 204 first-order planar gradiometers and 102 magnetometers, altogether 306 SQUID (superconducting quantum interference device) sensors. During the recordings, the subjects were seated under the helmet-shaped dewar in a magnetically shielded room. The recording passband was $0.1-173 \mathrm{~Hz}$, and the data were digitized at $603 \mathrm{~Hz}$. Eight minutes of raw data were recorded for each experiment. The vertical electrooculogram (EOG) was simultaneously recorded, and all traces coinciding with EOG activity exceeding $150 \mu \mathrm{V}$ or with signals in planar gradiometers exceeding $3000 \mathrm{fT} / \mathrm{cm}$ were excluded from the off-line averages.

Steady-state responses and source analysis. To obtain steady-state responses, 4000-6000 epochs were averaged with respect to the AM frequencies. In the binaural condition, SSFs elicited by 20.1 and $26.1 \mathrm{~Hz}$ AM tones overlapped in both hemispheres, and the responses were averaged separately time-locked to each AM frequency. The whole analysis period of two cycles, i.e., 77 and $100 \mathrm{msec}$ for the 26.1 and 20.1 $\mathrm{Hz}$ AMs, respectively, was used as the baseline for measuring amplitudes from signals digitally low-pass filtered at $33 \mathrm{~Hz}$.

Two equivalent current dipoles (ECDs) (Hämäläinen et al., 1993) were used to model the magnetic field distributions of SSFs. Previous studies have already shown that the auditory SSFs can be adequately explained by current sources in the auditory cortices of both hemispheres (Hari et al., 1989; Pantev et al., 1996; Gutschalk et al., 1999; Engelien et al., 2000;
Ross et al., 2000). First, single ECDs were found, at the peak latency on the strongest response to monaural stimuli, by a least-squares fit based on a subset of 54 planar gradiometers over each temporal area. The dipoles with goodness-of-fit values $\geq 80 \%$ were accepted for additional analysis; note that the selection of such an acceptance limit is rather arbitrary because the goodness-of-fit of the model depends, among other things, strongly on the number of channels chosen for the analysis (Hari et al., 1988). Then source waveforms were computed for the two-dipole model in which the dipoles, one in each hemisphere, were fixed in location and orientation, and the source strengths were calculated as the peak-to-peak values of the source waveforms. For binaural stimulation, the two fixed dipoles obtained for the responses to monaural stimuli were used to explain the measured signals.

Spectral analysis. Fast Fourier transform (FFT) spectra were calculated across 8192 samples of the continuously recorded MEG signals, and the FFT window was moved in steps of 4096 samples; this procedure resulted in frequency resolution of $0.074 \mathrm{~Hz}$. Approximately 70 spectra were averaged to improve the signal-to-noise ratio. The strengths of spectral peaks were measured at the modulation frequencies.

Statistical analysis. To compare the SSF amplitudes and spectral peaks between monaural and binaural conditions, the signal strengths during binaural stimulation were first calculated as percentage of the individual monaural signals. Paired $t$ tests were used in the statistical analysis of binaural suppression. Repeated-measures ANOVA, with hemisphere (left-right) and pathway (contralateral-ipsilateral) as the factors, and post hoc $t$ tests were performed.

\section{RESULTS}

Clear SSFs were observed at the AM frequencies over both temporal areas. Figure 1 shows the SSFs of one representative subject: the amplitude of the continuous monaural $1 \mathrm{kHz}$ tone was modulated at $20.1 \mathrm{~Hz}$ in the right ear and at $26.1 \mathrm{~Hz}$ in the left. The equivalent sources of the responses were located within the Sylvian fissures of both hemispheres, agreeing with activation of the supratemporal auditory cortices, as shown previously for many types of auditory SSFs (Romani et al., 1982; Hari et al., 1989; Pantev et al., 1996; Gutschalk et al., 1999; Engelien et al., 2000; Ross et al., 2000). Figure 1 also shows the source strengths as a function of time, demonstrating that the sources were weaker for binaural than monaural stimuli.

In spectral analysis, sharp peaks at AM frequencies were observed in both temporal areas. Figure 2 shows spectra of Subject 1 on one channel in the three conditions. Monaural sounds elicited sharp peaks at AM frequencies in both hemispheres, stronger in the contralateral than the ipsilateral hemisphere. These two spectral peaks were also seen during binaural listening, but then the amplitudes were suppressed. 
Left Hemisphere

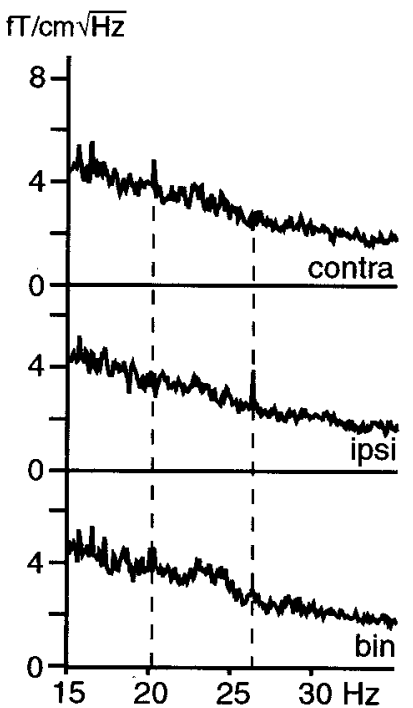

\section{Right Hemisphere}

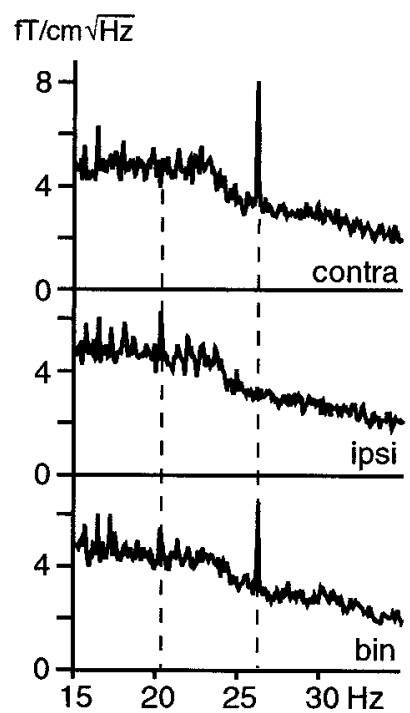

Figure 2. Single-channel spectra from both hemispheres of one subject in three conditions. The top row traces are responses to contralateral monaural stimulation (contra) $(26.1 \mathrm{~Hz} \mathrm{AM}$ to the left ear and $20.1 \mathrm{~Hz}$ $\mathrm{AM}$ to the right ear), the middle row shows responses to ipsilateral monaural stimulation (ipsi), and the bottom row shows responses to binaural stimulation (bin). The dashed vertical lines indicate the AM frequencies.

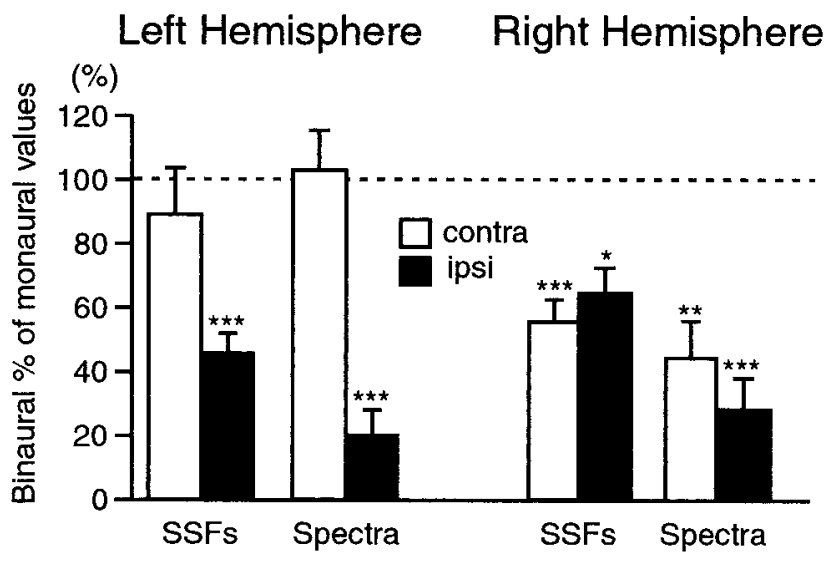

Figure 3. Mean SSF source strengths and spectral amplitudes during binaural listening expressed as percentages of responses to monaural stimulation (100\%) and shown separately for contralateral and ipsilateral inputs in both hemispheres. The error bars refer to the SEM values of 12 subjects $(* p<0.01 ; * * p<0.001 ; * * * p<0.0001)$.

Figure 3 shows the amplitudes of binaural responses as percentages of monaural responses in the whole group of subjects. In the left hemisphere, SSF source strengths to ipsilateral sounds were suppressed by $55 \%$ from those during monaural presentation ( $p<0.0001)$, whereas the change to contralateral sounds was not statistically significant. In the right hemisphere, the responses were symmetrically suppressed by 36 and $45 \%$ compared with contralateral and ipsilateral stimulations ( $p<0.0001$ for contralateral stimulation; $p=0.002$ for ipsilateral stimulation). ANOVA analyses showed a significant interaction between hemisphere and pathway ( $p=0.01)$, and the contralateral input was suppressed significantly ( $p=0.01)$ less than the ipsilateral input in the left but not in the right hemisphere.
The spectral analysis gave concordant results about the binaural interaction. In the left hemisphere, responses to ipsilateral sounds were only $19 \%(p<0.0001)$ of those during monaural presentation, whereas responses to contralateral sounds were not significantly affected. The right-hemisphere responses were symmetrically suppressed by 56 and $72 \%$ for contralateral and ipsilateral stimulations, respectively ( $p=0.0009$ for contralateral stimulation; $p<0.0001$ for ipsilateral stimulation). ANOVA analyses showed a significant interaction between hemisphere and pathway ( $p=0.002)$, and the contralateral input was suppressed significantly less than the ipsilateral input in the left hemisphere $(p<0.0001)$; both inputs were significantly suppressed in the right hemisphere $(p=0.0005)$.

In the control experiment with six subjects, the sides of AMs were reverted, and the results, obtained with $26.1 \mathrm{~Hz}$ AMs presented to the right ear and the $20.1 \mathrm{~Hz}$ AMs presented to the left ear, were practically identical to those in the main experiment. We are therefore confident that the obtained results did not drastically depend on the choice of the ear to which each AM was presented.

\section{DISCUSSION}

The impetus of the present work was the desire to label inputs from both ears so that they could be followed up to the level of the auditory cortex. This was successfully done by frequency tagging, i.e., by modulating the amplitude of the auditory inputs of each ear and by using the selectivity of MEG to signals arising in the auditory cortices of each hemisphere.

This procedure indicated clear binaural interaction, with hemispheric differences in the relative suppressions of contralateral versus ipsilateral inputs. In the left hemispheres of our righthanded subjects, responses to ipsilateral sounds were strongly suppressed during binaural listening, whereas responses to contralateral sounds were not significantly affected. In contrast, the right-hemisphere responses to both contralateral and ipsilateral ear presentations were symmetrically suppressed. Thus, the preference of the left hemisphere to right-ear input was accentuated during binaural hearing, agreeing with the frequently reported behavioral "right-ear and left-hemisphere advantage" of righthanded subjects during dichotic listening (Cowell and Hugdahl, 2000).

Analyses in time and frequency domains gave highly concordant results about the relative suppressions of the inputs from the two ears, indicating that either method could be used in assessing the contributions of monaural inputs to the binaural responses. However, because the phases of SSFs depend on the carrier frequency, modulation depth, and sound intensity (Ross et al., 2000), it might be difficult to obtain clear SSFs to AMs of complex sounds. Thus, high-resolution spectral analysis might be better suited for evaluation of binaural interaction during listening of music, speech, etc.

The frequency-tagging method introduced here might be valuable, e.g., in more detailed evaluation of reorganization of central auditory pathways observed in patients with unilateral deafness and long-lasting unilateral hearing impairment (Vasama et al., 1994; Vasama and Mäkelä, 1997; Fujiki et al., 1998). One reported change at the cortical level is the disappearance of clear contralateral dominance of the affected ear (Pelizzone et al., 1986; Scheffler et al., 1998; Bilecen et al., 2000). Thus, some inhibitory interactions that are operational during normal binaural hearing and that lead to competition of the auditory inputs might diminish in unilateral hearing-impaired patients. Such 
changes could most likely be detected with the frequency-tagging method. The method could also be applied to other conditions in which abnormal hemispheric balance is suspected, such as learning difficulties and some psychiatric disorders. Much basic work is, however, necessary to establish the stability of the findings and their dependence on both the stimulation parameters and the subject's state and task. Development of hemispheric dominance would be an interesting research topic as well; in the visual modality, frequency-tagging experiments have demonstrated weaker binocular suppression in infants than in adults (Brown et al., 1999).

We conclude that with MEG, combined with the frequencytagging method, contributions of each ear to the binaural responses in the auditory cortices of the two hemispheres can be readily assessed. Although we used pure tones as the carrier sounds, a similar procedure might be used to label, e.g., speech or music sounds, and thereby to assess the dominance or interaction of central auditory pathways during higher-order auditory processing.

\section{REFERENCES}

Bilecen D, Seifritz E, Radu EW, Schmid N, Wetzel S, Probst R, Scheffler K (2000) Cortical reorganization after acute unilateral hearing loss traced by fMRI. Neurology 54:765-767.

Brown RJ, Candy TR, Norcia AM (1999) Development of rivalry and dichoptic masking in human infants. Invest Ophthalmol Vis Sci 40:3324-3333.

Cowell P, Hugdahl K (2000) Individual differences in neurobehavioral measures of laterality and interhemispheric function as measured by dichotic listening. Dev Neuropsychol 18:95-112.

Engelien A, Schulz M, Ross B, Arolt V, Pantev C (2000) A combined functional in vivo measure for primary and secondary auditory cortices. Hear Res 148:153-160.

Fujiki N, Naito Y, Nagamine T, Shiomi Y, Hirano S, Honjo I, Shibasaki $\mathrm{H}$ (1998) Influence of unilateral deafness on auditory evoked magnetic field. NeuroReport 9:3129-3133.

Fujiki N, Jousmäki V, Hari R (2000) Binaural interaction revealed using frequency-tagged amplitude-modulated sounds. In: Biomag 2000, International Conference on Biomagnetism, Book of Abstracts, p 54. Espoo, Finland: Helsinki University of Technology.

Gutschalk A, Mase R, Roth R, Ille N, Rupp A, Hahnel S, Picton TW, Scherg M (1999) Deconvolution of $40 \mathrm{~Hz}$ steady-state fields reveals two overlapping source activities of the human auditory cortex. Clin Neurophysiol 110:856-868.

Hämäläinen M, Hari R, Ilmoniemi R, Knuutila J, Lounasmaa OV (1993) Magnetoencephalography-theory, instrumentation, and applications to noninvasive studies of the working human brain. Rev Mod Phys 65:413-497.

Hari R (1990) The neuromagnetic method in the study of the human auditory cortex. In: Auditory evoked magnetic fields and potentials.
Advances in audiology, Vol 6 (Grandori F, Hoke M, Romani G, eds), pp 222-282. Basel: Karger.

Hari R, Mäkelä JP (1988) Modification of neuromagnetic responses of the human auditory cortex by masking sounds. Exp Brain Res 71:87-92.

Hari R, Joutsiniemi SL, Sarvas J (1988) Spatial resolution of neuromagnetic records: theoretical calculations in a spherical model. Electroencephalogr Clin Neurophysiol 71:64-72.

Hari R, Hämäläinen M, Joutsiniemi SL (1989) Neuromagnetic steadystate responses to auditory stimuli. J Acoust Soc Am 86:1033-1039.

Imig TJ, Adrian HO (1977) Binaural columns in the primary field (A1) of cat auditory cortex. Brain Res 138:241-257.

Lins OG, Picton TW (1995) Auditory steady-state responses to multiple simultaneous stimuli. Electroencephalogr Clin Neurophysio 96:420-432.

Mäkelä JP, Ahonen A, Hämäläinen M, Hari R, Ilmoniemi R, Kajola M, Knuutila J, Lounasmaa OV, McEvoy L, Salmelin R, Salonen O, Sams M, Simola J, Tesche C, Vasama JP (1993) Functional differences between auditory cortices of the two hemispheres revealed by whole-head neuromagnetic recordings. Hum Brain Mapp 1:48-56.

Pantev C, Lütkenhöner B, Hoke M, Lehnertz K (1986) Comparison between simultaneously recorded auditory-evoked magnetic fields and potentials elicited by ipsilateral, contralateral and binaural tone burst stimulation. Audiology 25:54-61.

Pantev C, Roberts LE, Elbert T, Ross B, Wienbruch C (1996) Tonotopic organization of the sources of human auditory steady-state responses. Hear Res 101:62-74.

Pantev C, Ross B, Berg P, Elbert T, Rockstroh B (1998) Study of the human auditory cortices using a whole-head magnetometer: left vs. right hemisphere and ipsilateral vs. contralateral stimulation. Audiol Neurootol 3:183-190.

Patel AD, Balaban E (2000) Temporal patterns of human cortical activity reflect tone sequence structure. Nature 404:80-84.

Pelizzone M, Hari R, Mäkelä J, Kaukoranta E, Montandon P (1986) Activation of the auditory cortex by cochlear stimulation in a deaf patient. Neurosci Lett 68:192-196.

Reite M, Zimmerman JT, Zimmerman JE (1981) Magnetic auditory evoked fields: interhemispheric asymmetry. Electroencephalogr Clin Neurophysiol 51:388-392.

Reser DH, Fishman YI, Arezzo JC, Steinschneider M (2000) Binaural interactions in primary auditory cortex of the awake macaque. Cereb Cortex 10:574-584.

Romani GL, Williamson SJ, Kaufman L (1982) Tonotopic organization of the human auditory cortex. Science 216:1339-1340.

Ross B, Borgmann C, Draganova R, Roberts LE, Pantev C (2000) A high-precision magnetoencephalographic study of human auditory steady-state responses to amplitude-modulated tones. J Acoust Soc Am 108:679-691.

Scheffler K, Bilecen D, Schmid N, Tschopp K, Seelig J (1998) Auditory cortical responses in hearing subjects and unilateral deaf patients as detected by functional magnetic resonance imaging. Cereb Cortex $8: 156-163$.

Tiihonen J, Hari R, Kaukoranta E, Kajola M (1989) Interaural interaction in the human auditory cortex. Audiology 28:37-48.

Vasama JP, Mäkelä JP (1997) Auditory cortical responses in humans with profound unilateral sensorineural hearing loss from early childhood. Hear Res 104:183-190.

Vasama JP, Mäkelä JP, Parkkonen L, Hari R (1994) Auditory cortical responses in humans with congenital unilateral conductive hearing loss. Hear Res 78:91-97. 